\title{
Sarcocystis Attenuati N. Sp. (Apicomplexa: Sarcocystidae) Infecting the Asian Gray Shrew, Crocidura Attenuata (Insectivora: Soricidae), in China
}

junjie hu ( $\nabla$ jjhu@ynu.edu.cn )

Yunnan University https://orcid.org/0000-0002-3729-735X

Jun Sun

Yunnan University

Yanmei Guo

Kunming Medical University

Hongxia Zeng

Yunnan University

Yunzhi Zhang

Dali University

Jianping Tao

Yangzhou University

\section{Research Article}

Keywords: Crocidura attenuata, Sarcocystis attenuati, Life cycle, Morphological and molecular characterization

Posted Date: September 21st, 2021

DOI: https://doi.org/10.21203/rs.3.rs-907878/v1

License: (c) (i) This work is licensed under a Creative Commons Attribution 4.0 International License. Read Full License 


\section{Abstract}

Background: There are limited data on Sarcocystis in insectivores. The Asian gray shrew, Crocidura attenuata, is one of the most common species of insectivores in the family Soricidae distributed in South Asia and Southeast Asia. To date, Sarcocystis has never been recorded in this host.

Methods: Tissues from 42 Asian gray shrews were collected in China in 2017 and 2018. Sarcocysts were observed using light (LM) and transmission electron microscopy (TEM). To complete the parasite life cycle, muscle tissues of the host infected with sarcocysts were force-fed to two beauty rat snakes, Elaphe taeniura. Individual sarcocysts from different Asian gray shrews and oocysts/sporocysts isolated from the small intestines and feces of the experimental snakes were selected for DNA extraction, and seven genetic markers, including two nuclear loci (18S rDNA and ITS1), three mitochondrial genes (cox1, $\operatorname{cox} 3$ and $c y t b)$, and two apicoplastic genes $(r p o B$ and $c / p C)$, were amplified, sequenced and analyzed.

Results: Sarcocysts were found in 17 of 42 (40.5\%) Asian gray shrews. Under LM, the microscopic sarcocysts were exhibited saw-tooth-like protrusions measuring 3.3-4.5 $\mu \mathrm{m}$. Ultrastructurally, the sarcocyst wall contained numerous lancet- or leaf-like villous protrusions, similar to type $9 \mathrm{~h}$. The experimental beauty rat snakes shed oocysts/sporcysts measuring 11.9-16.7 × 9.2-10.6 $\mu \mathrm{m}$ with a prepatent period of 10 to 11 days. Comparing these sequences with those previously deposited in GenBank revealed that the $18 \mathrm{~S}$ rDNA sequences and $\operatorname{cox} 1$ sequences shared the highest similarity with those of $S$. scandentiborneensis recorded in tree shrews, Tuaia minor and T. tana (i.e., 97.6-98.3\% and $100 \%$ identity, respectively). Phylogenetic analysis based on 18 S rDNA, ITS1 or cox 1 sequences revealed that this parasite formed an independent clade with Sarcocystis spp. that utilize small animals as intermediate hosts and snakes as the known or presumed definitive host. On the basis of morphological and molecular characteristics and host specificity, the parasite was proposed as a new species, named $S$. attenuati.

Conclusions: Sarcocysts were recorded in Asian gray shrews for the first time. The sarcocysts were characterized morphologically and molecularly. The $18 \mathrm{~S}$ rDNA and cox 1 sequences of $S$. attenuati, named in the present study, shared the highest identities with those of $S$. scandentiborneensis. However, the sarcocysts of the two species of Sarcocystis were quite different under LM and TEM. Based on experimental infection, beauty rat snakes have been proven to be a definitive host of $S$. attenuati. As more species of Sarcocystis from insectivores and other small mammals are properly morphologically and molecularly characterized, we may gain a better understanding of the biodiversity, host specificity and evolution of Sarcocystis in the future.

\section{Background}

Species of the genus Sarcocystis (Apicomplexa: Sarcocystidae) exhibit an obligate two-host life cycle, with sexual development in the small intestine of the definitive host and asexual development in different tissues of the intermediate host, which are usually herbivores. More than 200 species of Sarcocystis have 
been described in a variety of wild or domesticated animals [1]. However, only two species, S. booliati and $S$. russuli, have been described and proposed to use insectivorous animals as intermediate hosts [1, $2,3]$. Insectivores are a group of mammals including 441 species and constitute almost $10 \%$ of extant mammal species. Asian gray shrew Crocidura attenuata, a species of insectivore in the family Soricidae, is one of the most common species throughout South and Southeast Asia [4]. To date, no species of Sarcocystis has been found and recorded in the host.

The present study describes the morphological and molecular characteristics of a new species of Sarcocystis detected in Asian gray shrews. Additionally, the life cycle of the new species was completed using animal experiments in the laboratory based on a speculation arising from phylogenetic analysis.

\section{Materials And Methods}

\section{Microscopic examination of sarcocysts from Asian gray shrews}

A total of 42 Asian gray shrews were captured by live trapping on farmland from July 2017 to December 2018 in Anning Prefecture, located in the mountainous zone of the central part of Yunnan Province, China. All hosts were killed with ether and transported to the zoological laboratory of Yunnan University, Kunming, China, on the same day.

Fresh preparations of the esophagus, diaphragm, tongue, skeletal muscles (thigh, loin, rump, and ribs) and heart of each animal were pressed and squeezed between two glass slides and inspected for sarcocysts using a stereomicroscope. Sarcocysts were extracted from muscular fibers using dissection needles and processed for light (LM) and transmission electron microscopy (TEM) and DNA analysis.

For TEM, the sarcocysts were fixed in $2.5 \%$ glutaraldehyde in cacodylate buffer $(0.1 \mathrm{M}, \mathrm{pH} 7.4)$ at 4 ${ }^{\circ} \mathrm{C}$ and postfixed in $1 \%$ osmium tetroxide in the same buffer, dehydrated in a graded alcohol series, and embedded in Durcupan. Ultrathin sections were stained with uranyl acetate and lead citrate and examined using a JEM100-CX transmission electron microscope at $80 \mathrm{kV}$.

\section{Experimental infection of potential definitive hosts}

To complete the life cycle of the parasite, three beauty rat snakes, Elaphe taeniura, purchased from a pet market in Kunming city, were housed separately in steel cages at ambient temperature and humidity. The snakes were force-fed quail eggs, and the feces of the snakes were examined for two weeks via the flotation technique to confirm that they were coccidian free.

Pieces of muscle from a wild-caught Asian gray shrew were force-fed to two beauty rat snakes, and the remaining snake was kept as a control. Before inoculation, it was confirmed by extensive microscopic examination that the muscles of the Asian gray shrew fed to experimental snakes only contained sarcocysts of the new species. The experimental snakes were each fed muscle pieces containing approximately 300 sarcocysts, and fecal samples of the snakes were examined daily for two weeks postinfection $(\mathrm{PI})$ via the flotation method to determine the presence of oocysts/sporocysts. All snakes 
were killed 29 days PI. The small intestine of each snake was removed and digested $\left(1 \%\right.$ trypsin, $37^{\circ} \mathrm{C}, 3$ hours), and the digested tissue was filtered and centrifuged (5000 r/min, $10 \mathrm{mins}$ ). The sediments were tested to determine the presence of oocysts/sporocysts using light microscopy. Oocysts/sporocysts from the feces and small intestines of the experimental snakes were collected and stored in sterile water at 4 ${ }^{\circ} \mathrm{C}$ for DNA extraction.

\section{Molecular characterization}

A total of 11 samples, including ten individual sarcocysts separated from different Asian gray shrews and oocysts/sporocysts (approximately 250) collected from the experimental snakes, were subjected to genetic DNA extraction using the TIANamp Genomic DNA Kit (Tiangen Biotech Ltd., Beijing, China) according to the manufacturer's instructions. The sarcocysts and oocysts/sporocysts were characterized on the basis of seven genes, including two nuclear loci (18S rDNA and ITS1), three mitochondrial genes $(c o x 1, \operatorname{cox} 3$ and $c y t b)$, and two apicoplastic genes $(r p o B$ and $c / p C)$. The primers used to amplify these genes are shown in Table 1.

PCR was performed in a PCR cocktail with a total volume of $25 \mu$ l that included $12.5 \mu \mathrm{l}$ Green Taq Mix

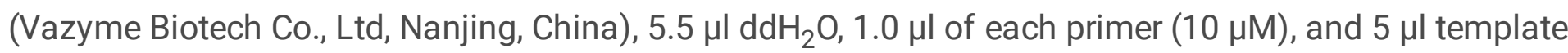
DNA. The cycling parameters differed for each gene. For 18S rDNA and ITS1, the cycling parameters started with denaturation at $94^{\circ} \mathrm{C}$ for 5 mins, followed by 35 cycles of $94^{\circ} \mathrm{C}$ for $1 \mathrm{~min}, 57^{\circ} \mathrm{C}$ for $1 \mathrm{~min}$, and $72{ }^{\circ} \mathrm{C}$ for 1.5 mins, with a final extension at $72{ }^{\circ} \mathrm{C}$ for 10 mins. For $\operatorname{cox} 1$ and $\operatorname{cox} 3$, the cycling parameters started with denaturation at $94^{\circ} \mathrm{C}$ for 5 mins, followed by 35 cycles of $94{ }^{\circ} \mathrm{C}$ for 1 min, 54 ${ }^{\circ} \mathrm{C}$ for $1 \mathrm{~min}$, and $72{ }^{\circ} \mathrm{C}$ for 1.5 mins, with a final extension at $72{ }^{\circ} \mathrm{C}$ for 7 mins. For $c y t b, r p o B$ and $c / p C$, the cycling parameters started with denaturation at $94^{\circ} \mathrm{C}$ for 5 mins, followed by 35 cycles of $94{ }^{\circ} \mathrm{C}$ for 1 min, $52{ }^{\circ} \mathrm{C}$ for $1 \mathrm{~min}$, and $72{ }^{\circ} \mathrm{C}$ for 1.5 mins, with a final extension at $72{ }^{\circ} \mathrm{C}$ for 7 minutes. The PCR products were purified, cloned, sequenced, and assembled using the methods described in our previous paper [9].

At present, there are limited or few nucleotide sequences of the $c o x 3, c y t b, r p o B$ and $c / p C$ genes of Sarcocystis species deposited in GenBank. Therefore, in the present study, only 18S rDNA, ITS1 and cox1 sequences of the new species were used to infer the relationship with other Sarcocystis spp.

using MEGAX software [10]. Maximum parsimony (MP) trees for the three genes were created with a treebisection-regrafting (TBR) algorithm. The reliability of the MP phylograms was tested via the bootstrap method using 1,000 replications. The 18S rDNA sequences of Sarcocystis spp. from different hosts were downloaded from GenBank and aligned with the ClustalW program implemented in MEGAX, and the alignment was subsequently checked visually; some sequences were slightly truncated at both ends so that all sequences started and ended at the same nucleotide positions (i.e., 101 and 1,828, respectively) of the sequence of $S$. cruzi (JX679468). The final alignment consisted of a total of 43 nucleotide sequences and 1,865 aligned positions of 39 taxa. Cystoisospora ohioensis (GU292304), Besnoitia besnoiti (DQ227418), Hammondia heydorni (GQ984224), and Toxoplasma gondii (U03070) were chosen as outgroups. 
A total of 44 ITS1 sequences of 31 taxa used in the analysis were aligned with the ClustalW program integrated in MEGAX. Some sequences were truncated at both ends so that all sequences started and stopped at the same nucleotide positions (i.e., 1,671 and 2,477, respectively) of the sequence of $S$. zuoi (KU341120). The final alignment consisted of 1,924 aligned positions. Toxoplasma gondii (KM657806) and $H$. triffittae (KJ396594) were used as outgroup species to root the tree.

In total, 31 mitochondrial $\operatorname{cox} 1$ sequences of 27 taxa used in the analysis were aligned with the ClustalW program integrated in MEGAX. Some sequences were slightly truncated at both ends so that all sequences started and stopped at the same nucleotide positions (i.e., 48 and 1,020, respectively) of the $S$. cruzi sequence (KT901095). The final alignment consisted of 992 aligned positions with no gaps. Toxoplasma gondii (JX473253), H. heydorni (JX473251) and H. triffittae (JX473247) were used as outgroup species to root the tree.

\section{Results}

\section{LM and TEM observations of sarcocysts}

Spindle-shaped sarcocysts were found in 17 of 42 (40.5\%) Asian gray shrews, located in skeletal muscle, the esophagus, the diaphragm, the tongue and the heart. Only one form of sarcocysts was observed. The observation of fresh samples at the light microscopy level showed that the cyst walls of the sarcocysts had numerous, 3.3-4.5 $\mu \mathrm{m}$-long $(\mathrm{n}=25)$ saw-tooth-like protrusions (Fig. 1a). Mature sarcocysts were $740-1,355 \times 117-250 \mu \mathrm{m}$ in size; they were septate and contained bradyzoites measuring 8.2-10.4 $\times$ $2.0-3.0 \mu \mathrm{m}(\mathrm{n}=40)$ in size.

Ultrastructurally, the sarcocysts had lancet- or leaf-like villous protrusions measuring 2.8-6.4 $\times 0.4-0.9$ $\mu \mathrm{m}(\mathrm{n}=15)$, which contained numerous electron-dense granules in the core; microtubules or fibrils were absent. The primary cyst wall had minute undulations over the entire sarcocyst surface, lined by an electron-dense layer. The nearly vertical protrusions were separated from each other at inconsistent distances. A layer of ground substance measuring 1.2-1.6 $\mu \mathrm{m}(\mathrm{n}=15)$ in thickness was located immediately beneath the primary sarcocyst wall (Fig. 1b).

\section{Infection of the definitive host}

The two beauty rat snakes fed muscle tissue containing sarcocysts in an Asian gray shrew excreted sporulated oocysts/sporocysts (Fig. 1C, d) in their feces, one beginning on Day 10 and another on Day $11 \mathrm{PI}$. Upon the death of the snakes at Day $29 \mathrm{PI}$, numerous oocysts/sporocysts were also observed within the small intestines of the experimental animals. Under light microscopy, the sporulated oocysts measured 11.9-16.7 × 9.2-10.6 $\mu \mathrm{m}$ (average $13.5 \times 9.9 \mu \mathrm{m})(\mathrm{n}=27)$, with two elliptical sporocysts measuring $9.2-10.6 \times 6.3-6.8 \mu \mathrm{m}$ (average $9.9 \times 6.6 \mu \mathrm{m})(\mathrm{n}=30)$.

No oocysts/sporocysts were found in the feces or small intestine of the control snake.

\section{Molecular analysis}


Seven genes (18S rDNA, ITS1, cox1, cox3, cytb, rpoB and $c / p C$ ) were successfully amplified from ten individual sarcocysts and oocysts/sporocysts. A total of 16 clones, including ten from each sarcocyst and six from oocysts/sporocysts, were sequenced, assembled, and submitted to GenBank. The ten $18 \mathrm{~S}$ rDNA sequences obtained from the sarcocysts were 1,863-1,867 bp in length and shared $99.7-100 \%$ identity (average $99.8 \%$ ). The six $18 \mathrm{~S}$ rDNA sequences obtained from the oocysts/sporocysts were $1,865-1,868$ bp in length and shared $99.7-100 \%$ identity (average $99.8 \%$ ). Therefore, only three sequences from sarcocysts (accession numbers MZ826981-MZ826983) and two sequences from oocysts/sporocysts (MZ826984 and MZ826985) were deposited in GenBank. The sequence identity between the sarcocysts and oocysts/sporocysts was $99.7-100 \%$, with an average of $99.8 \%$. The most similar sequences in GenBank were those of S. scandentiborneensis (MN733816 and MN733817) obtained from the tree shrews Tuaia minor and T. tana (ranging 97.6-98.3\% identity, on average 97.9\%), followed by S. zuoi (ranging 96.7-98.0\% identity, average 97.2\%) from the Malayan field rat, Rattus tiomanicus (KU341118-KU341121); Sarcocystis sp. (AB251613) from the raccoon Procyon lotor (ranging from 95.9-96.9\% identity, average 96.3\%); and S. clethrionomyelaphis (KP057504, KF309700, and KF309701) from the large oriental vole, Eothenomys miletus (ranging from 95.9-96.4\% identity, average $96.2 \%)$.

The ten ITS1 sequences obtained from sarcocysts were 874-978 bp in length and shared $97.9-100 \%$ identity (average $99.0 \%$ ). The six ITS1 sequences obtained from oocysts/sporocysts were $875-877$ bp in length and shared $98.6-100 \%$ identity (average $99.0 \%$ ). Therefore, only 14 sequences, including nine from sarcocysts (MZ826986-MZ826994) and five (MZ826995-MZ826999) from oocysts/sporocysts, were deposited in GenBank. The similarity between the sarcocysts and oocysts/sporocysts was 97.4$100 \%$, with an average of $98.7 \%$. The most similar sequences in GenBank were those of $S$. zuoi (KU341118-KU341121) from the Malayan field rat, but the identity was only $72.4-86.0 \%$ (average $76.4 \%)$.

The ten cox 1 sequences obtained from sarcocysts were 1,333 bp in length and shared $99.8-100 \%$ identity (average 99.9\%). The six cox1 sequences obtained from oocysts/sporocysts were $1333 \mathrm{bp}$ in length and shared $99.9-100 \%$ identity (average 99.9\%). Therefore, only five cox 1 sequences, including three from sarcocysts (MZ889669-MZ889671) and two from oocysts/sporocysts (MZ889672 and MZ889673), were deposited in GenBank. The similarity between the sarcocysts and the oocysts/sporocysts was $99.8-100 \%$, with an average of $99.9 \%$. The most similar sequences in GenBank were those of S. scandentiborneensis (MN732561 and MN732562, 100\% identity), followed by those of Sarcocystis sp. (MT411016, 99.8\% identity) from the greater white-toothed shrew, Crocidura russula; and S. canis (KX721496 and KX721497) from the Indo-Pacific bottlenose dolphin, Tursiops aduncus (ranging 95.0-95.4\% identity, average $95.2 \%)$.

The 16 cox3 sequences from the sarcocysts and the oocysts/sporocysts were $675 \mathrm{bp}$ in length and were completely identical. Therefore, only one sarcocyst sequence (OK001462) and one oocyst/sporocyst sequence (OK001463) were deposited in GenBank. No sequences with significant similarity to these sequences were found in GenBank. 
The 16 cytb sequences from the sarcocysts and the oocysts/sporocysts were 1,080 bp in length and were completely identical. Therefore, only one sarcocyst sequence (OK001464) and one oocyst/sporocyst sequence (OK001465) were deposited in GenBank. The most similar sequences in GenBank were those of S. falcatula (MF034168-MF034187) from the budgerigar, Melopstittacus undulatus, and the identity was $96.4 \%$.

The $16 r p o B$ sequences obtained from the sarcocysts and the oocyst/sporocysts were $511 \mathrm{bp}$ in length and shared $100 \%$ identity. Therefore, only one sarcocyst sequence (OK001466) and one oocysts/sporocysts sequence (OK001467) were deposited in GenBank. The most similar sequence in GenBank was that of S. neurona (GQ851961) obtained from the southern sea otter, Lutris nereis, and the identity was $91.9 \%$.

The $16 c / p C$ sequences from the sarcocysts and the oocysts/sporocysts were $534 \mathrm{bp}$ in length and shared $100 \%$ identity. Therefore, only one sarcocyst sequence (OK001468) and one oocyst/sporocyst sequence (OK001469) were deposited in GenBank. The most similar sequences were those of $S$. falcatula (KP871717) and S. neurona (KP871716), and the identity was $92.5 \%$.

\section{Phylogenetic analysis}

Phylogenetic analysis based on $18 \mathrm{~S}$ rDNA, ITS1, or $\operatorname{cox} 1$ sequences confirmed that the parasite found in the present study belonged to Sarcocystis (Figs. 2-4). In the phylogenetic tree inferred from 18S rDNA sequences (Fig. 2), the parasite formed an individual clade and clustered with Sarcocystis spp. using colubrids as definitive or presumed definitive hosts (i.e., S. scandentiborneensis (MN733816), S. zuoi (KU341120), S. clethrionomyelaphis (KP057504) and Sarcocystis sp. ex Procyon lotor(AB251613)). These species belonged to a group containing Sarcocystis spp. with pythons as definitive hosts (i.e., Sarcocystis sp. ex Morelia viridis (KC201639), S. zamani (KU244524) and S. singaporensis (AF434054)). In the phylogenetic tree inferred from ITS1 sequences, the parasite formed an individual group and clustered with Sarcpcystis spp. with sankes as definitive or presumed definitive hosts (i.e., S. zuoi (KU341120), S. zamani (KU244525), Sarcocystis sp. (MH590230) and S. singaporensis (KU341123)). In the phylogenetic tree inferred from cox 1 sequences (Fig. 4), the parasite formed an individual clade with S. scandentiborneensis (MN732562).

Based on the morphological characteristics of sarcocysts, molecular analysis and host specificity, a new species name, Sarcocystis attenuati, is proposed for the organism found in Asian gray shrews from Anning Prefecture, China.

\section{Taxonomic summary of Sarcocystis attenuati n. sp.}

Diagnosis: The sarcocysts were microscopic, 740-1,355 $\mu \mathrm{m}$ long, and 117-250 $\mu \mathrm{m}$ wide. The thickwalled sarcocysts had 3.3-4.5 $\mu \mathrm{m}$-long saw-tooth-like protrusions. The sarcocysts were divided by septa into a series of internal compartments filled with bradyzoites measuring 8.2-10.4 $\times 2.0-3.0 \mu \mathrm{m}$. TEM revealed sarcocysts with lancet- or leaf-like protrusions measuring 2.8-7.4 $\times 0.4-0.9 \mu \mathrm{m}(\mathrm{n}=15)$, which 
contained electron-dense granules in the core. The nearly vertical protrusions were separated from each other at inconsistent distances, similar to wall type $9 \mathrm{~h}$ classified by Dubey et al. (2016).

Type (natural) intermediate host: Asian gray shrew, Crocidura attenuata.

Type locality: Anning Prefecture $\left(25^{\circ} 24^{\prime} \mathrm{N}, 102^{\circ} 35^{\prime} \mathrm{E}\right.$; altitude 1,894 m), China.

Site of infection: Muscular tissues throughout the body, including the heart.

Experimental definitive host: beauty rat snake, Elaphe taeniura.

Prevalence: Sarcocysts were found in 17 of 42 (40.5\%) examined Asian gray shrews.

Etymology: Species named after the species of its intermediate host.

Molecular characterization: Nucleotide sequences of the 18S rDNA (MZ826981-MZ826985), ITS1 (MZ826986-MZ826999), cox1 (MZ889669-MZ889673), coX3 (OK001462 and OK001463), cytb (OK001464 and OK001465), rpoB (OK001466 and OK001467), and clpC(OK001468 and OK001469) of the new species have been deposited in GenBank. According to $18 \mathrm{~S}$ rDNA sequences, $S$. attenuati may be differentiated from $S$. scandentiborneensis obtained from lesser or large tree shrews. However, the cox 1 sequences are unsuitable for differentiating it from S. scandentiborneensis or even Sarcocystis sp. obtained from the greater white-toothed shrew.

Specimens deposited: Formalin-fixed tissues containing cysts of $S$. attenuati as well as photomicrographs obtained from the LM and TEM examination of the sarcocysts were deposited at the Zoological Specimen Museum of Yunnan University, Kunming, China (collection number Pro2018004).

\section{Remarks}

Insectivores are an abundant group of small mammals comprising hedgehogs, moonrats, shrews, and moles. Publications regarding Sarcocystis spp. in insectivores are very limited. To our knowledge, sarcocysts have been observed only in several species of insectivores, including the shrew mole Urotrichus talpoides in Japan [11], moonrat, Echinosorex gymnurus, in Malaysia [2], the short-tailed shrew Blarina brevicauda in the USA [12], the white-toothed shrew Crocidura russula in Russia [3], and the common shrew Sorex araneus in Lituanica [13]. Unfortunately, the descriptions of most sarcocysts recorded in the abovementioned cases are not detailed. Based on the morphological characterization of sarcocysts, only two species, $S$. boollati and $S$. russuli, have been proposed, which were found in moonrat and white-toothed shrews, respectively. With the aid of LM, it was shown that both species exhibit smooth, thin sarcocyst walls $(<1 \mu \mathrm{m})[2,3]$. Ultrastructurally, $S$. booliati sarcocysts show small knob-like protrusions, similar to wall type $1 \mathrm{~b}$ [14]. In the present study, S. attenuati n. sp. found in Asian gray shrews presented a thick sarcocyst wall (3.3-4.5 $\mu \mathrm{m}$ in length), similar to wall type $9 \mathrm{~h}$, which was unambiguously different from those of $S$. boollati and $S$. russuli. 
The ultrastructure of sarcocysts has traditionally been used as a reliable indicator for the characterization of different Sarcocystis species in a given host. Nucleotide sequence analysis has now been suggested to be a more useful tool for the delineation or identification of Sarcocystis from the same or different hosts. However, different genetic markers have revealed different levels of intra- or interspecific sequence diversity [15-17]. Here, seven molecular markers (18S rDNA, ITS1, cox1, cox3, cytb, rpoB, and $c / p C$ ) were sequenced and characterized in DNA samples from $S$. attenuati. The analysis showed that the sequences of these seven genes of the parasite presented high intraspecific similarities (i.e., $99.7-100 \%, 97.9-100 \%$, $99.8-100 \%, 100 \%$, and $100 \%$, and $100 \%, 100 \%$, respectively). The comparison of these sequences with those previously deposited in GenBank showed that the $18 \mathrm{~S}$ rDNA and cox 1 sequences of $S$. attenuati shared the highest identity with those of S. scandentiborneensis (i.e., 97.6-98.3\% and 100\%, respectively). Sarcocystis scandentiborneensis occurs in lesser or large tree shrews belonging to the family Tupaiidae collected from Malaysia. With the aid of LM, the cyst wall of $S$. scandentiborneensis was shown to present tightly packed figure-like protrusions (2-10 $\mu \mathrm{m}$ in length) that can assume a brushlike appearance; ultrastructurally, the tightly packed figure-like protrusions contain bundled microtubules that extend into the ground substance [18], similar to wall type $11 \mathrm{~b}$ or 12 according to the classification of Dubey et al. (2016) [1]. Additionally, the cox 1 sequences of $S$. attenuata shared a high similarity of $99.8 \%$ with that of Sarcocystis sp. obtained from the greater white-toothed shrew collected from Spain. However, sarcocysts of the greater white-toothed shrew have not been described [19].

The phylogenetic relationships among the majority of analyzed Sarcocystis spp. suggest their coevolution with their definitive hosts rather than their intermediate hosts [20]. The phylogenetic trees based on 18S rDNA sequences and ITS1 sequences revealed that $S$. attenuati formed an individual clade with Sarcocystis spp. from small mammals using snakes as definitive or putative definitive hosts, showing especially close relationships with $S$. scandentiborneensis, Sarcocystis sp. ex Procyon lotor, $S$. zuoi, and S. clethrionomyelaphis.

The infection experiment confirmed the speculation arising from the phylogenetic analysis and proved that beauty rat snakes can serve as an experimental definitive host of $S$. attenuati. The beauty rat snake is native to eastern and southeastern Asia and feeds mainly on shrews and rodents, although the consumption of amphibians, reptiles, and birds by this species has also been reported [21]. To date, only two species of Sarcocystis, S. zuoi from Norway rat, R. norvegicus, and S. clethrionomyelaphis from large oriental vole, E. miletus, have been proven to use species of Elaphe as definitive hosts via transmission experiments $[22,23]$. Morphologically, sarcocysts of $S$. zuoi exhibit sloping finger-like protrusions, and the base of the protrusions is highly branched $[22,24]$, similar to wall type 17; the sarcocysts of $S$. clethrionomyelaphis present thin highly folded protrusions, which often bend along the cyst surface [23], similar to type wall type $10 \mathrm{f}$. Therefore, the sarcocysts of $S$. attenuati can be easily morphologically differentiated from those of $S$. zuoi and $S$. clethrionomyelaphis. It is very common that one predator acts as the definitive host of more than one species of Sarcocystis. For example, Python reticulatus is the definitive host of three species of Sarcocystis in rats (i.e., S. singaporensis, S. villivillosi, and S. zamani) [25]. 


\section{Conclusions}

In summary, this report demonstrates the presence of Sarcocystis that form microscopic cysts in Asian gray shrews. Based on the observed morphological characteristics and host specificity, a new species name, $S$. attenuati, is proposed for the new species infecting Asian gray shrews. This is the first record of Sarcocystis in this host. Seven genetic markers, 18S rDNA, ITS1, cox1, cox3, cytb, rpoB, and $c / p C$, of the species were sequenced and characterized. Interestingly, the $18 \mathrm{~S}$ rDNA sequences and cox 1 sequences of the species shared the highest similarities with those of $S$. scandentiborneensis obtained from tree shrews, reaching $100 \%$ identity for cox 1 . However, the sarcocysts of the two species presented different morphological characteristics according to LM and TEM observations. Experimental infection revealed that beauty rat snake can serve as the experimental definitive host of $S$. attenuati, which was also proven by the high similarities of the nucleotide sequences of the seven genes between the sarcocysts and oocysts/sporocysts of S. attenuati. As more species of Sarcocystis from different insectivores and other small mammals are properly morphologically and molecularly characterized, we may gain a better understanding of the biodiversity, host specificity and evolution of Sarcocystis in the future.

\section{Abbreviations}

LM: light microscopy; TEM: transmission electron microscopy; PCR: polymerase chain reaction; 18 S rDNA: 18 S ribosomal DNA; ITS1: intergenic transcribed spacer region 1; cox1: cytochrome oxidase subunit 1 ; cox3: cytochrome oxidase subunit 3 ; cytb: cytochrome b; rpoB: RNA polymerase beta subunit; $c / p C$. casyenolitic protease C; MP: maximum parsimony; TBR: tree-bisection-regrafting.

\section{Declarations}

\section{Ethics approval and consent to participate}

The present study was approved by the Animal Ethics Committee of Yunnan.

University (permission number ynucae20180002). All experimental animals were handled following good animal practices according to the regulations for the Care and Use of Laboratory Animals of the National Institutes of Health, China.

\section{Consent for publication}

Not applicable.

\section{Availability of data and materials}

The datasets used and/or analysed during the current study are available from the corresponding author on reasonable request. 
The authors declare that they have no competing interests.

\section{Funding}

This study was supported by the Natural Sciences Foundation of China (Grant

31460557).

\section{Authors' contributions}

JH conceived the study, analyzed the data, and drafted the manuscript. JS, YG and ZH conducted the molecular experiments and the experimental infection. $Y Z$ collected the specimens and recorded prevalence and morphological observations. JT analyzed and interpreted some of the results. JS and YG contributed equally to this study and share first authorship with $\mathrm{JH}$.

\section{Acknowledgments}

Not applicable.

\section{References}

1. Dubey JP, Calero-Bernal R, Rosenthal BM, Speer CA, Fayer R. Sarcocystosis of animals and humans. 2nd ed. Boca Raton: CRC Press; 2016.

2. Dissanaike AS, Poopalachelvam M. Sarcocystis booliatin. sp. and a parasite of undetermined taxonomic position, Octoplasma garnhami n. gen. n. sp., from the moonrat, Echinosorex gymnurus. Southeast Asian J Trop Med Public Health. 1975;6:175-85.

3. Pak SM, Sklyarova ON, Dymkova ND. Sarcocysts (Sporozoa, Apicomplexa) of some wild mammals. Izvest Akad Nauk Kazakh Ser Biol. 1991;5:35-40. (In Russian).

4. Zhang RZ. Zoogeography of China. Beijing: Science Press; 1999. (In Chinese).

5. Barta JR, Martin DS, Liberator PA, Dashkevicz M, Anderson JW, Feighner SD, et al. Phylogenetic relationships among eight Eimeria species infecting domestic fowl inferred using complete small subunit ribosomal DNA sequences. J Parasitol. 1997;83(2):262-71.

6. Fischer S, Odening K. Characterization of bovine Sarcocystis species by analysis of their $18 \mathrm{~S}$ ribosomal DNA sequences. J Parasitol. 1998;84(1):50-4.

7. Fenger CK, Granstrom DE, Langemeier JL, Stamper S, Donahue JM, Patterson JS, et al. Identification of opossums (Didelphis virginiana) as the putative definitive host of Sarcocystis neurona. J Parasitol. 1995;81(6):916-9.

8. Sercundes MK, Valadas SY, Keid LB, Oliveira TM, Ferreira HL, Vitor RW, et al. Molecular phylogeny of Toxoplasmatinae: comparison between inferences based on mitochondrial and apicoplast genetic sequences. Rev Bras Parasitol Vet. 2016;25(1):82-9. 
9. Hu JJ, Liu TT, Liu Q, Esch GW, Chen JQ, Huang S, et al. Prevalence, morphology, and molecular characteristics of Sarcocystis spp. in domestic goats (Capra hircus) from Kunming China. Parasitol Res. 2016;115(10):3973-81.

10. Kumar S, Stecher G, Li M, Knyaz C, Tamura K. MEGA X: Molecular Evolutionary Genetics Analysis across Computing Platforms. Mol Biol Evol. 2018;35(6):1547-49.

11. Ohbayashi M, Masegi T, Kubota K. Parasites of the Japanese shrew mole. Jap J Vet Res. 1972;20:50-6.

12. Huffman JE, Roscoe DE. The occurrence of a sporozoan in the myocardium of short-tailed shrew (Blarina brevicauda). J Wildl Dis. 1981;17(2):209-12.

13. Grikieniené J, Mažeikyté R. Investigation of sarcosporidians (Sarcocystis) of small mammals in Kamasta landscape reserve and its surroundings. Acta Zool Litu. 2000;10(3):55-68.

14. Kan SP, Dissanaike AS. Ultrastructure of Sarcocystis booliati Dissanaike and Poopalachelvam, 1975 from the moonrat, Echinosorex gymnurus, in Malaysia. Inter J Parasitol. 1976;6(4):321-6.

15. Gjerde B. Phylogenetic relationships among Sarcocystis species in cervids, cattle and sheep inferred from the mitochondrial cytochrome c oxidase subunit I gene. Int J Parasitol. 2013;43:579-91.

16. Huang ZM, Ye YL, Zhang HZ, Deng SS, Tao JJ, Hu JJ, et al. Morphological and molecular characterizations of Sarcocystis miescheriana and Sarcocystis suihominis in domestic pigs (Sus scrofa) in China. Parasitol Res. 2019;118(12):3491-6.

17. Pan J, Ma C, Huang Z, Ye Y, Zeng H, Deng S, et al. Morphological and molecular characterization of Sarcocystis wenzeli in chickens (Gallus gallus) in China. Parasit Vectors. 2020;13(1):512.

18. Ortega Pérez P, Wibbelt G, Brinkmann A, Galindo Puentes JA, Tuh FYY, Lakim MB, et al. Description of Sarcocystis scandentiborneensis sp. nov. from treeshrews (Tupaia minor, T. tana) in northern Borneo with annotations on the utility of $\mathrm{COI}$ and $18 \mathrm{~S}$ rDNA sequences for species delineation. Int $\mathrm{J}$ Parasitol Parasites Wildl. 2020;12:220-31.

19. Fernández-Escobar M, Millán J, Chirife AD, Ortega-Mora LM, Calero-Bernal R. Molecular survey for cyst-forming coccidia (Toxoplasma gondii, Neospora caninum, Sarcocystis spp.) in Mediterranean periurban micromammals. Parasitol Res. 2020;119(8):2679-86.

20. Dolezel D, Koudela B, Jirků M, Hypsa V, Oborník M, Votýpka J, et al. Phylogenetic analysis of Sarcocystis spp. of mammals and reptiles supports the coevolution of Sarcocystis spp. with their final hosts. Int J Parasitol. 1999;29(5):795-8.

21. Zhao EM, Huang MH, Zong Y. Fauna Sinica. Reptilia, Volume 3, Squamata, Serpentes. Beijing: Sciences Press; 1998.

22. Hu JJ, Meng Y, Guo YM, Liao JY, Song JL. Completion of the life cycle of Sarcocystis zuoi, a parasite from the Norway rat, Rattus norvegicus. J Parasitol. 2012;98(3):550-3.

23. Hu JJ, Liu TT, Liu Q, Esch GW, Chen JQ. Sarcocystis clethrionomyelaphis M. 1986 (Apicomplexa: Sarcocystidae) infecting the large oriental vole Eothenomys miletus (Thomas) (Cricetidae: Microtinae) and its phylogenetic relationships with other species of Sarcocystis Lankester, 1882. Syst Parasitol. 2015;91(3):273-9. 
24. Hu JJ, Ma TC, Li XR. A new species of sarcocysts (Sporozoa, Eucoccoddoda) from Rattus norvegicus. Acta Zootaxonomica Sinica. 2005;30(2):287-90. (in Chinese).

25. Häfner U, Frank W. Host specificity and host range of the genus Sarcocystis in three snake-rodent life cycles. Zentralbl Bakteriol Mikrobiol Hyg A. 1984;256:296-9.

\section{Tables}

Table 1 Primers used for the amplification of seven DNA regions

\begin{tabular}{|c|c|c|c|}
\hline DNA region & Primer name & Primer sequence $₫ 5^{\prime}-3^{\prime} \rrbracket$ & Reference \\
\hline \multirow[t]{4}{*}{ 18S rDNA } & ERIB1 ${ }^{\mathrm{a}}$ & ACCTGGTTGATCCTGCCAG & [5] \\
\hline & $\mathrm{S} 2^{\mathrm{b}}$ & CTGATCGTCTTCGAGCCCCTA & [6] \\
\hline & $S 3^{\mathrm{a}}$ & TTGTTAAAGACGAACTACTGCG & [6] \\
\hline & $\mathrm{B}^{\mathrm{b}}$ & GATCCTTCTGCAGGTTCACCTAC & [7] \\
\hline \multirow[t]{2}{*}{ ITS1 } & ITSFa & GTTCCGGTGAATTATTCGGACTGTT & This study \\
\hline & $\mathrm{ITSR}^{\mathrm{b}}$ & GATGATTCCCTGAATTCTGCAATTC & This study \\
\hline \multirow[t]{2}{*}{$\operatorname{cox} 1$} & $526 \mathrm{~F} 1^{\mathrm{a}}$ & TCCTTCCTGGCGTACAACAATCAT & This study \\
\hline & $1209 \mathrm{R} 1^{\mathrm{b}}$ & GGGGCATGACATTGAAAGCAAGTA & This study \\
\hline \multirow[t]{2}{*}{$\operatorname{cox} 3$} & $\operatorname{cox} 3 \mathrm{~F} 1^{\mathrm{a}}$ & GCTTTAAACGTATTGTATTTCAATA & This study \\
\hline & $\operatorname{COX} 3 \mathrm{R} 1^{\mathrm{b}}$ & TCAACCATAGACATTCTATGAAATG & This study \\
\hline \multirow[t]{2}{*}{ cytb } & $1080 \mathrm{CYTBF}^{\mathrm{a}}$ & ATGAGTTTAGTGCGAGCACATTT & This study \\
\hline & 1080 CYTBR $^{\text {b }}$ & TTAATATAGACATACAGCTAAGCTTGTGA & This study \\
\hline \multirow[t]{2}{*}{ гров } & RpoBFa & ATTTTTGTGGATATGATTTTGAAGATGC & [8] \\
\hline & $\mathrm{RpoBR}^{\mathrm{b}}$ & ТTТССАТАТСТTССАСАТААТTТАТСТС & [8] \\
\hline \multirow[t]{2}{*}{$c l p C$} & ClpCF-1 ${ }^{a}$ & GGAGCACCACCTGGGTATGT & This study \\
\hline & ClpCR-1 $1^{b}$ & CGAGCTCCATATAAAGGATGATAAG & This study \\
\hline
\end{tabular}

${ }^{\mathrm{a}}$ Forward primer $\mathrm{X}^{\mathrm{b}}$ Reverse primer. The primers of ITS1, $\operatorname{cox} 1, \operatorname{cox} 3, c y t b$, and $c / p C$ used in the study were designed using OLIGO 5.0 (National BioScience, Plymouth, Minnesota, USA) based on the highly conserve areas of corresponding sequences for Sarcocystis spp., Toxoplasma gondii, Besnoitia besnoiti and Hammondia heydorni deposited in GenBank. 


\section{Figures}
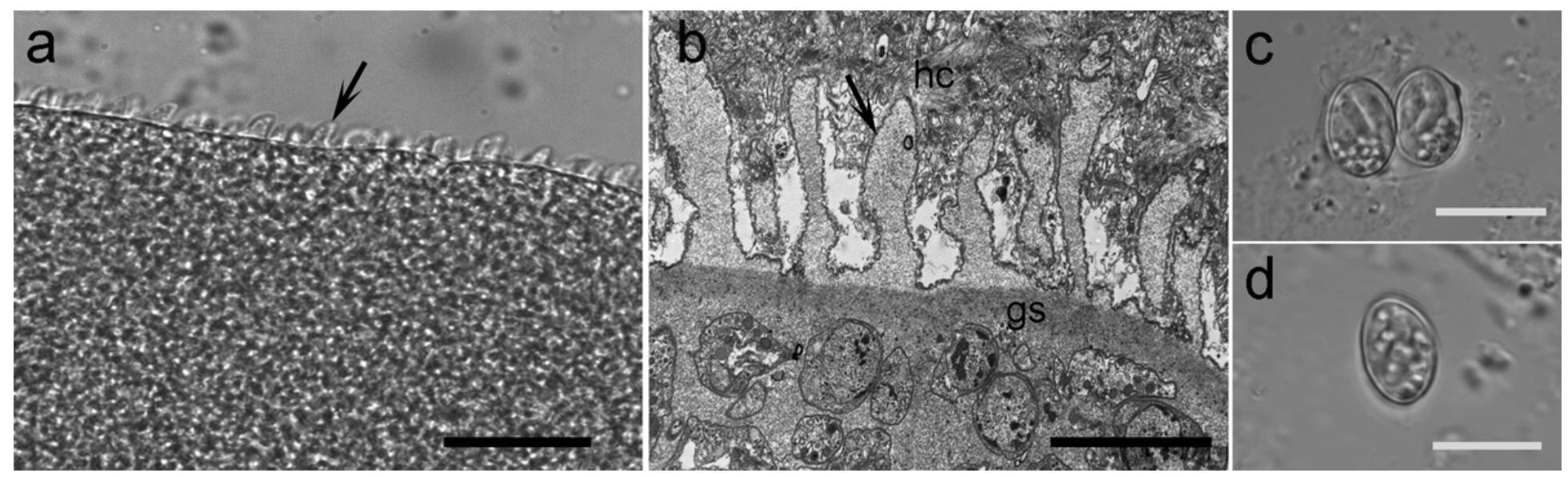

\section{Figure 1}

Stages of Sarcocystis attenuati n. sp. in Asian gray shrew, Crocidura attenuata. a Light micrograph (LM) of cysts of S. attenuati (unstained). Sarcocyst bounded by a wall with numerous saw-tooth-like villous protrusions (arrow); b Transmission electron micrograph of the sarcocyst wall of S. attenuati. Sarcocyst surrounded by host wall (hc), and the primary cyst wall containing numerous lancet- or leaf-like villous protrusions (arrow). The ground substance (gs) beneath the protrusions was fine grained; c LM image showing oocysts and sporocysts of $\mathrm{S}$. attenuati in the feces of experimentally infected beauty rat snakes, Elaphe taeniura. Scale-bars: a, $20 \mu \mathrm{m} ; \mathrm{b}, 5 \mu \mathrm{m} ; \mathrm{c}, 10 \mu \mathrm{m}, \mathrm{d} 10 \mu \mathrm{m}$. 


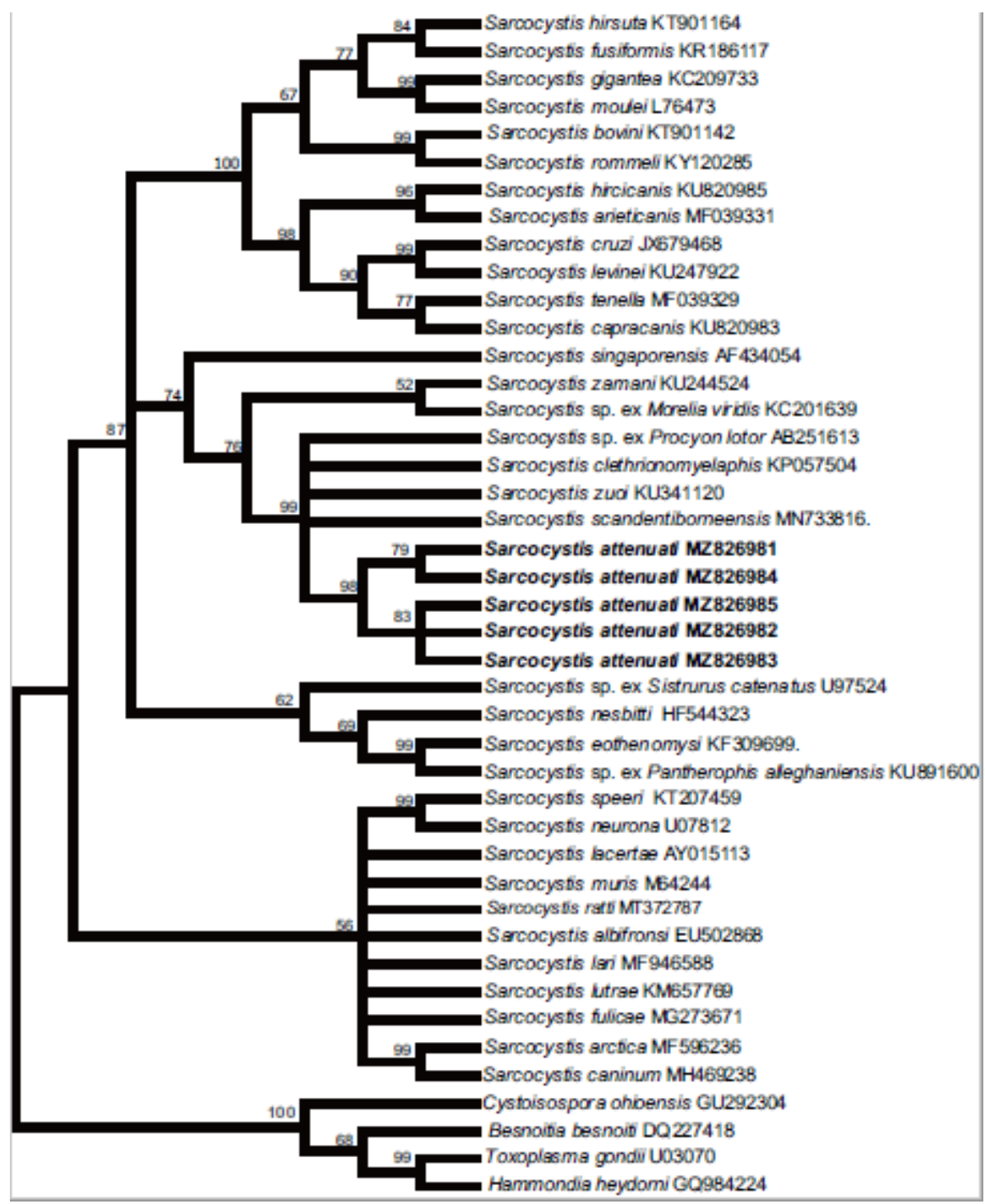

Figure 2

Phylogenetic tree of selected members of Sarcocystidae based on 18S rDNA sequences, inferred using the maximum parsimony method with the tree-bisection-regrafting algorithm. GenBank accession numbers of all sequences included in the analysis are given behind the taxon names. The values between the branches represent percent bootstrap value per 1,000 replicates. Bootstrap values below $50 \%$ are not shown. The five sequences of Sarcocystis attenuati (GenBank MZ826981- MZ826985, shown in bold) group within the Sarcocystidae and form a clade with other Sarcocystis spp. with snakes as definitive hosts. 


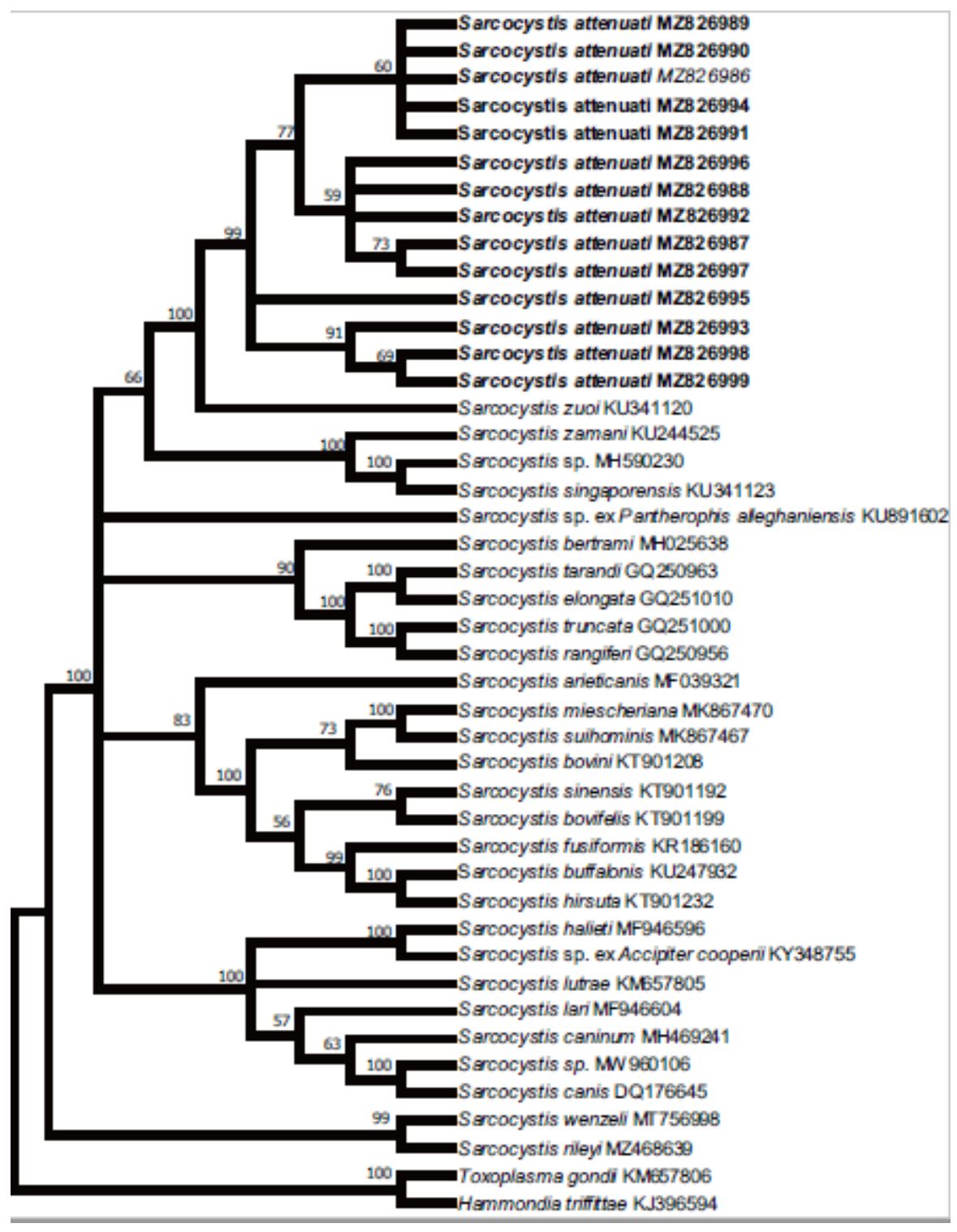

Figure 3

Phylogenetic tree of selected members of Sarcocystidae based on ITS1 sequences, inferred using the maximum parsimony method with the tree-bisection-regrafting algorithm. GenBank accession numbers of all sequences included in the analysis are given behind the taxon names. The values between the branches represent percent bootstrap value per 1,000 replicates. Bootstrap values below $50 \%$ are not shown. The 14 sequences of Sarcocystis attenuati (GenBank MZ826986-MZ826999, shown in bold) group within the Sarcocystidae and form a clade with other Sarcocystis spp. with snakes as definitive hosts. 


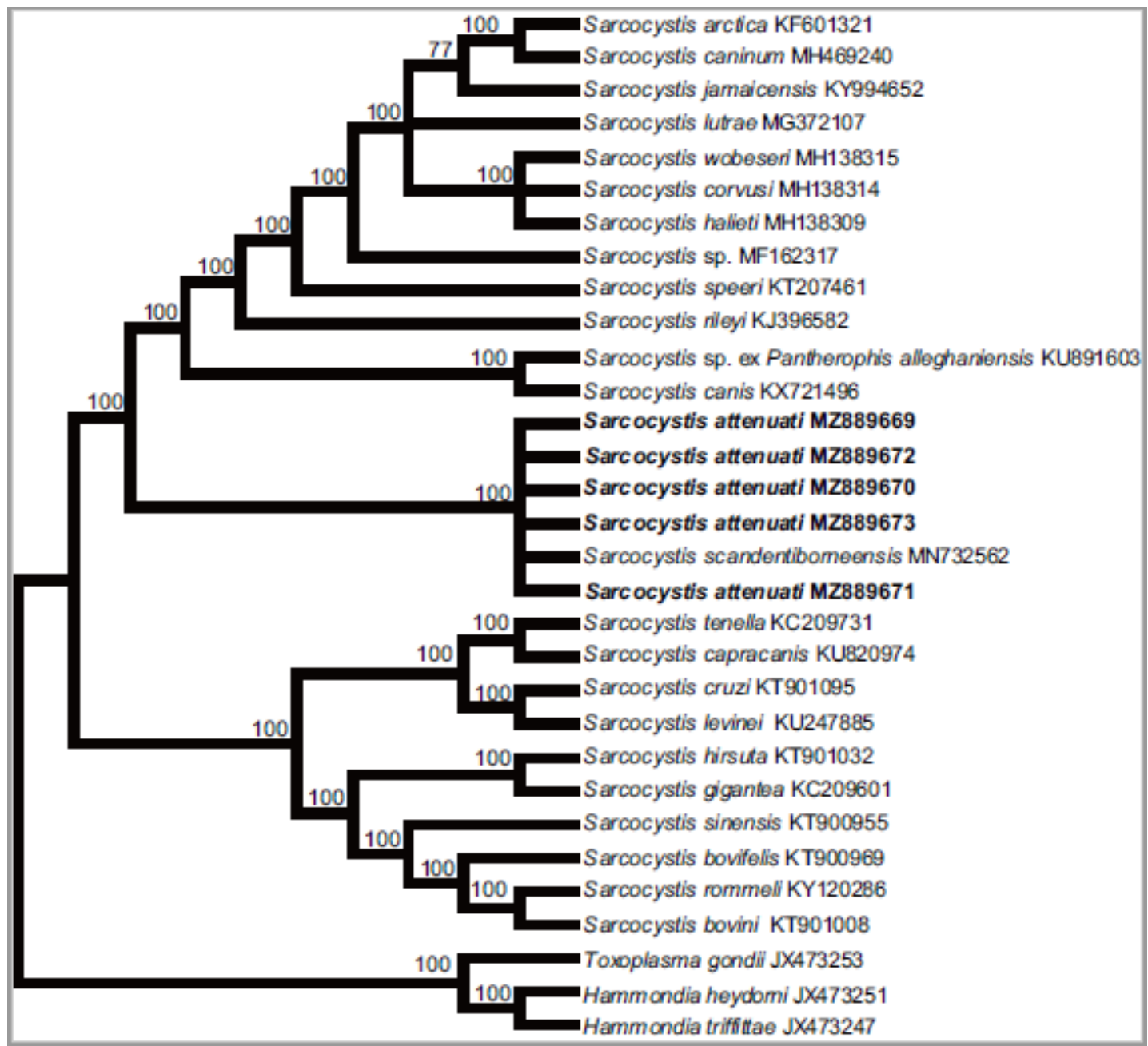

Figure 4

Phylogenetic tree of selected members of Sarcocystidae based on partial mitochondrial cox 1 sequences, inferred using the maximum parsimony method. GenBank accession numbers of all sequences included in the analysis are given behind the taxon names. The values between the branches represent percent bootstrap value per 1,000 replicates. Bootstrap values below $50 \%$ are not shown. The seven sequences of Sarcocystis attenuati (GenBank MZ889669-MZ889673, shown in bold) firmed formed a clade with S. scandentiborneensis.

\section{Supplementary Files}

This is a list of supplementary files associated with this preprint. Click to download.

- Graphicabstractimage.jpg 